\title{
Seminare / Séminaires / Seminari
}

Obwohl rund 40\% aller Ärztinnen und Ärzte früher oder später eine eigene Arztpraxis führen und nebst dem medizinischen Know-how auch jenes eines Unternehmers innehaben sollten, wird im Verlauf des Medizinstudiums die Thematik der Unternehmensführung nie oder nur kaum fokussiert. Die FMH Services, welche sich um die betriebswirtschaftlichen Angelegenheiten der FMH-Mitglieder kümmert, schliesst diese Lücke und bietet dazu spezifische Seminare an. In diesen Seminaren wird den Ärztinnen und Ärzten Grundlagen vermittelt für den Einstieg in die eigene Arztpraxis/Gruppenpraxis, deren Führung und deren optimalen Übergabe an einen/eine Nachfolger/in. Die Ärztinnen und Ärzte werden auf den Umgang und die Zusammenarbeit mit Behörden sowie Fachspezialisten, wie Treuhänder, Versicherungs- und Finanzberater, Kreditberater bei Banken, Juristen, Architekten, usw. vorbereitet. Die einzelnen Themen werden von ausgewiesenen Experten der FMH und der FMH Services referiert.

\section{Praxiseröffnung}

\section{Teilnehmende}

Das Seminar richtet sich an Ärztinnen und Ärzte, welche vor einer Praxiseröffnung oder einer Praxisübernahme stehen.

\section{Themen}

Juristische Aspekte (Praxisbewilligung, Zulassung zur Sozialversicherung, Vertragswesen usw.), TARMED (Überblick über neues Tarifsystem und dessen Anwendung), Praxiseinrichtung (Raumplanung, Inneneinrichtung, Kostenberechnung), Praxisadministration (Personalwesen, Leistungserfassungs- und Abrechnungssysteme, Praxismarketing), Finanzierung einer Arztpraxis (Businessplan, Kreditarten, Absicherungsmöglichkeiten), Versicherungen (Personen- und Sachversicherungen rund um die Arztpraxis).

\section{Unterlagen}

Aufbauend auf einer Checkliste zur Praxiseröffnung enthält der Seminarordner, welcher jedem/jeder Teilnehmer/in abgegeben wird, wichtige Basisinformationen zu den behandelten Themen.

\section{Kosten / Sponsoren}

Das Seminar ist für FMH-Mitglieder gratis.
Die Kosten werden von folgenden Sponsoren gedeckt: Versicherung der Schweizer Ärzte, Schweizerische Ärzte-Krankenkasse, Druckerei Schmid-Mogelsberg, www.daCapo.ch und Axon Lab AG.

\section{Daten}

\begin{tabular}{|c|c|c|}
\hline \multirow[t]{2}{*}{ Nr. 02} & \multicolumn{2}{|l|}{ Donnerstag, 5. Juni 2003} \\
\hline & Bern $\quad 9.00-16.30 \mathrm{Uhr}$ & Hotel Bern \\
\hline \multirow[t]{2}{*}{ Nr. 03} & \multicolumn{2}{|l|}{ Donnerstag, 4. September 2003} \\
\hline & Zürich $\quad 9.00-16.30 \mathrm{Uhr}$ & FMT Zürich \\
\hline \multirow[t]{2}{*}{ Nr. 04} & \multicolumn{2}{|c|}{ Donnerstag, 6. November 2003} \\
\hline & Bern $\quad 9.00-16.30 \mathrm{Uhr}$ & Hotel Kreuz \\
\hline
\end{tabular}

\section{Anmeldung}

Sie können sich bei nachfolgend aufgeführter Adresse (FMH Consulting Services) oder via Internet unter www.fmhservices.ch einschreiben.

\section{Praxisübergabe/-übernahme}

\section{Teilnehmende}

Das Seminar richtet sich an zukünftige Praxisübergeber/innen sowie Praxisübernehmer/innen.

\section{Themen}

Juristische Aspekte (Übergabe der Krankengeschichten, Praxisübernahmevertrag, Mietvertrag usw.), Praxiseinrichtung (Aus- und Umbaumöglichkeiten, Detailkonzept, Kostenberechnungen), Versicherungen (Übergabe/Übernahme von Versicherungsverträgen, Vorsorgeplanung usw.), Bewertung einer Arztpraxis (Berechnung Inventarwert und Goodwill), Steuern (Steuerfolgen aus Sicht des Praxisübergebers sowie des Praxisübernehmers, wie z.B. Liquidations- und Grundstückgewinnsteuer).

\section{Unterlagen}

Die Seminarteilnehmer/innen erhalten einen Seminarordner mit praxisorientierten Unterlagen zum Thema Praxisübergabe/-übernahme.

\section{Kosten / Sponsoren}

Das Seminar ist für FMH-Mitglieder gratis.

Die Kosten werden von folgenden Sponsoren gedeckt: Versicherung der Schweizer Ärzte und Schweizerische Ärzte-Krankenkasse. 
Daten

\begin{tabular}{|c|c|c|c|}
\hline \multirow[t]{2}{*}{ Nr. 06} & \multicolumn{3}{|c|}{ Donnerstag, 12. Juni 2003} \\
\hline & Bern & $9.30-16.30 \mathrm{Uhr}$ & Hotel Bern \\
\hline \multirow{2}{*}{ Nr. 07} & \multicolumn{3}{|c|}{ Donnerstag, 11. September 2003} \\
\hline & Zürich & $9.30-16.30 \mathrm{Uhr}$ & FMT Zürich \\
\hline \multirow[t]{2}{*}{ Nr. 08} & \multicolumn{3}{|c|}{ Donnerstag, 13. November 2003} \\
\hline & Bern & $9.30-16.30 \mathrm{Uhr}$ & Hotel Bern \\
\hline
\end{tabular}

\section{Anmeldung}

Sie können sich bei nachfolgend aufgeführter Adresse (FMH Consulting Services) oder via Internet unter www.fmhservices.ch einschreiben.

\section{Buchhaltung und Steuern}

\section{Teilnehmende}

Das Seminar richtet sich an Praxiseröffner/innen, Praxisübernehmer/innen sowie an bereits praxistätige Ärztinnen und Ärzte.

\section{Themen}

Im Buchhaltungsteil erfahren die Seminarteilnehmer/innen, welche Massnahmen vor der Praxiseröffnung getroffen werden müssen und welches deren organisatorischen Voraussetzungen sind. Es wird aufgezeigt, wie eine Doppelte Buchhaltung geführt wird und sie erhalten Einblick in eine EDV-unterstützte Buchführungslösung. Im Steuerteil werden die Methoden der Steuerplanung aus versicherungstechnischer sowie aus treuhänderischer Sicht aufgezeigt.

\section{Unterlagen}

Die Seminarteilnehmer/innen erhalten einen Seminarordner mit Unterlagen zu den behandelten Themen.

\section{Kosten / Sponsoren}

Das Seminar ist für FMH-Mitglieder gratis.

Die Kosten werden von folgendem Sponsor gedeckt: Salzmann MEDICO

\section{Daten}

\begin{tabular}{|c|c|c|}
\hline \multirow[t]{2}{*}{ Nr. 10} & \multicolumn{2}{|l|}{ Donnerstag, 26. Juni 2003} \\
\hline & Bern $\quad 9.30-16.30 \mathrm{Uhr}$ & Hotel Kreuz \\
\hline \multirow[t]{2}{*}{ Nr. 11} & \multicolumn{2}{|c|}{ Donnerstag, 18. September 2003} \\
\hline & Zürich $\quad 9.30-16.30 \mathrm{Uhr}$ & FMT Zürich \\
\hline \multirow[t]{2}{*}{ Nr. 12} & \multicolumn{2}{|c|}{ Donnerstag, 20. November 2003} \\
\hline & Bern $\quad 9.30-16.30 \mathrm{Uhr}$ & Hotel Bern \\
\hline
\end{tabular}

\section{Anmeldung}

Sie können sich bei nachfolgend aufgeführter Adresse (FMH Consulting Services) oder via Internet unter www.fmhservices.ch einschreiben.

\section{Praxiscomputer-Workshop}

\section{Teilnehmende}

Der Workshop richtet sich an praxiseröffnende sowie an bereits praxistätige Ärztinnen und Ärzte.

\section{Inhalt}

Die Seminarteilnehmer/innen erhalten die Gelegenheit, die Praxisadministrationssoftware von sechs EDV-Unternehmen kennenzulernen. Sie erhalten einen Einblick in TARMED-kompatible Lösungen für die Leistungsabrechnung, die Führung elektronischer Krankengeschichten, die Einbindung von Laborgeräten und von Finanzbuchhaltungslösungen. Bei den anwesenden Firmen handelt es sich jeweils um fünf Hersteller von EDVGesamtlösungen in der Arztpraxis sowie um eine Abrechnungskasse.

\section{Kosten}

Der Workshop ist für FMH-Mitglieder gratis.

\section{Daten}

Nr. 14 Donnerstag, 3. Juli 2003 Bern 14.00-17.30 Uhr BEA Bern Expo

\section{Folgende Softwareanbieter können Sie kennenlernen}

\section{Ärztekasse, Urdorf ZH (MediWin CB Pro)}

Als Marktleader seit 1964 im Bereich Praxisadministration können Sie bei der Ärztekasse auf eine ganzheitliche Lösung für Ihre Fragen betreffend Abrechnungsvarianten, Computerwahl, Netzwerke, Formular- und Briefbearbeitung usw. zählen. Die statistischen Erhebungen (im Auftrag der FMH, Roko) sind ein anerkanntes betriebswirtschaftliches und standesorganisatorisches Hilfsmittel für die moderne Praxisführung. Nebst innovativen Ideen und Lösungen ist uns eine umfassende Kundenbetreuung ein Anliegen. Unsere Standorte befinden sich in Basel, Bern, Genf, Luzern, Le Landeron, Lugano, St. Gallen und Zürich. Sie definieren Ihre Wünsche - wir bieten Ihnen die Lösung!

\section{Delemed AG, Gümligen (delemed ${ }^{\circledR} P E X$ II)}

Delemed AG entwickelt und vertreibt seit 12 Jahren erfolgreich PEX II, die einfache, sympathische und effiziente Software für die Arztpraxis und Kliniken. Durch einen modularen Aufbau kann das System jederzeit den aktuellen Bedürfnissen des Kunden angepasst werden. Dank unserer Vielseitigkeit im medizinischen Umfeld und unserem Kundenstamm von über 550 Anwendern sind wir ein optimale Partner für Sie. 
Kern Concept, Gossau (Aeskulap)

Aeskulap ist ein extrem schnelles Windows-Programm, das zusammen mit einer SQL-Datenbank höchste Verarbeitungsgeschwindigkeit, Kompaktheit und schnellste Zugriffsmöglichkeiten gewährleistet. Aeskulap bietet für alle Bedürfnisse eine optimale Lösung: vom einfachen Abrechnungssystem mit sämtlichen Leistungserfassungsmöglichkeiten bis zur vollelektronischen, papierlosen KG-Führung. Aeskulap ist ein modular aufgebautes, anpassbares System mit einer Vielzahl von Modulen. Wir sind in den letzten sieben Jahren von 4 auf 24 Mitarbeiter/innen gewachsen, mit über 800 Systeminstallationen mit einem Total von über 1100 Kunden.

Praxidata AG, Thun-Allmendingen (Praxidata) Praxidata AG ist eines der führenden Systemhäuser für Medizininformatik und gehört seit Frühjahr 2002 zur Triamun Gruppe. Praxidata steht für Sicherheit, Innovation und gute Lösungen, womit ein reibungsloser Ablauf im Praxisalltag gewährleistet ist. Mit den Praxidata-Modulen wurde eine wirtschaftliche Komplettlösung geschaffen, die sich den unterschiedlichen Bedürfnissen in den Arztpraxen optimal anpasst. Im Hinblick auf TARMED hat Praxidata mit der Neuentwicklung der Produktepalette auf NET-Technologie von Microsoft für Ihre Kunden wiederum eine gute Lösung gefunden. Damit stellt das Unternehmen der Triamun Gruppe jenseits herkömmlicher 32bit-Lösungen den Anschluss an die webbasierte Technologiegeneration sicher.

VarioSoft D. Cohen, Bern (mFmed EVOLUTION) Variosoft konzentriert sich ausschliesslich auf den Ärztebereich. Benutzerfreundlichkeit, Effizienz, Ergonomie und Flexibilität sind nur einige der Stärken unserer echten 32-Bit-Applikation mFmed EVOLUTION.

Vitodata AG, Ohringen b. Winterthur (vitoMed2) Die Vitodata AG ist seit 1980 im Markt tätig und konzentriert sich auf Ärzte- und Kliniklösungen. Die Unternehmung zeichnet sich durch die innovative Haltung aus und eröffnet laufend neue Einsatzgebiete - immer mit dem erwünschten Nutzen für die Benutzer. Die Vitodata AG war die erste Unternehmung im Markt mit der kostenlos integrierten Krankengeschichte. Diese Entwicklung wurde vorangetrieben und hat heute einen
Stand erreicht, der sich deutlich von Mitbewerbern absetzt - vor allem im fachlich medizinischen Bereich. Als Marktführer Schweiz ist es für die Vitodata AG eine Verpflichtung, im Sinne des Investitionsschutzes für den Käufer den Fortbestand $\mathrm{zu}$ sichern und unternehmerisch stets korrekt zu handeln.

\section{Anmeldung}

Sie können sich bei nachfolgend aufgeführter Adresse (FMH Consulting Services) oder via Internet unter www.fmhservices.ch einschreiben.

\section{Patientengerechtes Verhalten in der Arztpraxis}

\section{Teilnehmende}

Das Seminar richtet sich an das Medizinische Praxispersonal sowie an Ärztinnen und Ärzte.

\section{Inhalt}

Der erste Eindruck einer Patientin bzw. eines Patienten, welchen sie bzw. er von der Praxis vermittelt bekommt, ist für den Praxiserfolg sehr wichtig. Den Seminarteilnehmern/-innen wird aufgezeigt, wie dem Patienten ein «Kundengefühl» vermittelt wird und wie Reklamationen erledigt werden. Es wird darauf eingegangen, welches die Möglichkeiten des Telefons, als akustische Visitenkarte, und des persönlichen Patientenkontakts sind.

\section{Kosten}

Für Mitglieder des Schweizerischen Verbandes Medizinischer Praxisassistentinnen Fr. 285.-

Für übrige Teilnehmer/innen (MPA ohne Verbandsmitgliedschaft, Ärztin/Arzt) Fr. 300.-

\section{Daten}

\begin{tabular}{|c|c|c|}
\hline \multirow[t]{2}{*}{ Nr. 16} & Donnerstag, 22. Mai 2003 & \\
\hline & Zürich $\quad 9.00-17.45 \mathrm{Uhr}$ & FMT Zürich \\
\hline \multirow[t]{2}{*}{ Nr. 17} & Donnerstag, 30. Oktober 2003 & \\
\hline & Bern $\quad 9.00-17.45 \mathrm{Uhr}$ & Hotel Bern \\
\hline
\end{tabular}

\section{Anmeldung}

Sie können sich bei nachfolgend aufgeführter Adresse (FMH Consulting Services) oder via Internet unter www.fmhservices.ch einschreiben. 


\section{Ouverture et gestion d'un cabinet médical}

\section{Participants}

Le séminaire est destiné aux médecins sur le point d'ouvrir ou de reprendre un cabinet médical.

\section{Contenu}

La réalisation d'un cabinet médical ne s'improvise pas. Vous allez dépendre du financement et de la gestion de votre cabinet médical. Ce séminaire traitera de l'environnement socio-politique qui sera le vôtre en tant qu'indépendant, et vous permettra de vous y préparer. Les thèmes suivants seront développés lors de ce séminaire: l'aménagement, le financement, les assurances, la fiscalité, la comptabilité et l'administration de votre cabinet.

\section{Documentation}

Basé sur une check-liste pour l'ouverture d'un cabinet médical, le classeur remis à chaque participant contient toutes les informations importantes.

\section{Frais / Sponsors}

Le séminaire est gratuit pour les membres FMH. Les frais sont couverts par les sponsors suivants: Caisse-Maladie des Médecins Suisses et Axon Lab SA.

\section{Date}

No 19 Jeudi 18 septembre 2003 Hostellerie Genève $9.00-17.00 \mathrm{~h}$ de la Vendée

\section{Inscription}

Vous pouvez vous inscrire à l'adresse mentionnée ci-dessous ou via internet www.fmhservices.ch

\section{Remise/reprise d'un cabinet médical}

\section{Participants}

Le séminaire s'adresse aux médecins désirant remettre ou reprendre un cabinet médical.

\section{Contenu}

Questions principalement abordées: explication des aspects juridiques de la remise et de la reprise d'un cabinet médical (contrat de remise de cabinet, transfert des dossiers médicaux, etc.), mesures architecturales à envisager et coût de cellesci, questions d'assurance à mettre au point de part et d'autre (par exemple planification de la prévoyance, reprise de contrats d'assurance), estimation de la valeur du cabinet (valeur d'inventaire et goodwill) et conséquences fiscales d'une remise ou d'une reprise de cabinet (moment idéal de remise, impôt sur la liquidation et le bénéfice de l'immeuble, etc.).

\section{Documentation}

Les participants du séminaire reçoivent un classeur et les documents contenant des conseils pratiques au sujet de la remise ou de la reprise d'un cabinet médical.

\section{Frais / Sponsor}

Le séminaire est gratuit pour les membres FMH. Les frais sont couverts par le sponsor suivant: Caisse-Maladie des Médecins Suisses.

\section{Date}

$\begin{array}{lll}\text { No } 21 & \text { Jeudi } 6 \text { novembre } 2003 & \text { Hostellerie } \\ \text { Genève } \quad 9.00-16.30 \text { h } & \text { de la Vendée }\end{array}$

\section{Inscription}

Vous pouvez vous inscrire à l'adresse mentionnée ci-dessous ou via internet www.fmhservices.ch

\section{Cours d'introduction à I'ordinateur}

\section{Participants}

Le cours s'adresse aux médecins sur le point d'ouvrir un cabinet médical ou aux médecins déjà établis.

\section{Contenu}

L'exposé d'introduction informe les participants sur les applications informatiques disponibles pour le cabinet médical. Les participants peuvent ensuite se familiariser avec des logiciels concernant la gestion du cabinet médical, proposés par quatre entreprises différentes qui leur proposent un aperçu des programmes les plus performants. Ils seront ainsi à même de choisir la solution informatique la plus appropriée et de savoir quelles sont les erreurs à éviter.

\section{Frais}

Le cours est gratuit pour les membres FMH.

\section{Date}

No 23 Jeudi 25 septembre 2003 Beaulieu Lausanne 14.00-17.30 h Exploitation SA 


\section{Les entreprises suivants vous pouvez connaître}

Caisse des Médecins, Thônex (Mediwin CB Pro) 40 ans d'existence, et 14 ans de facturation par PC, la Caisse des Médecins a su profiter de l'expérience acquise pour développer des produits adaptés aux besoins réels du cabinet médical. Ses logiciels dont la dernière version a été particulièrement développée pour le TARMED ont depuis longtemps intégré la facturation électronique des données et obtenu le label "programme gestion confiance» délivré par la commission informatique de l'AMG. L'implantation régionale est, en plus, un atout supplémentaire pour le cabinet médical.

\section{BB-Soft, Lausanne (MédiACT)}

Créé en 1988 et dirigé par un médecin non praticien, BB-Soft a conçu et réalisé MédiACT avec une idée directrice: votre confort. Sur Macintosh ou sur PC (Windows), grande facilité d'usage et rapidité d'apprentissage. Agréé comme Programme-Confiance par l'Asscociation des Médecins de Genève.

\section{GNT, Gespower New Technologies,}

\section{Chêne-Bourg (MEDISOFT 5 Tarmed)}

GNT développe des applications dans le domaine médical, médecins et laboratoires d'analyses. Nos produits sont commercialisés à l'échelle européenne. Médisoft 5 Tarmed est un logiciel complet, puissant et convivial. Il répond totalement aux besoins d'un cabinet médical de 1 à 50 médecins. Modernité et richesse de fonctionnalités à faible coût. Médisoft 5 Tarmed est un programme de confiance de la commission informatique de l'AMG.

\section{PSIPI SA, Plan-les-Ouates /GE (PsiMed)}

PSIPI SA est spécialisée dans le développement de logiciels médicaux: gestion de cabinet médical, dossier médical électronique, imagerie, connexions aux appareils médicaux, échanges électroniques entre médecins, laboratoires, hôpitaux, bases de données externes. Extrême souplesse de paramétrisation des applications et facilité d'utilisation. Synergie entre les produits et les services PSIPI SA et d'autres acteurs du domaine de la santé: médicaments, assurances, communications sécurisées. PsiMed est agréé programme de confiance de l'Association des Médecins de Genève.

\section{Inscription}

Vous pouvez vous inscrire à l'adresse mentionnée ci-contre ou via internet www.fmhservices.ch

\section{Contabilità e fiscalità (Ticino)}

\section{Contenuto}

L'obiettivo di questo seminario è di dare al medico le conoscenze necessarie per utilizzare autonomamente lo strumento contabile e per operare con più efficienza ed economicità, nonché far comprendere al medico il significato della pianificazione fiscale.

Mediante esempi pratici elaborati in gruppo, verrà illustrato in maniera semplice ma completa il sistema di gestione della contabilità di uno studio medico con effetto anche sulla pianificazione fiscale.

\section{Spese}

$\begin{array}{ll}\text { Membri ordinari o straordinari FMH } & \text { fr. 150.- } \\ \text { Per accompagnatori } & \text { fr. } 75 .-\end{array}$

\section{Date}

No 25 Giovedì 13 novembre 2003 FMH Services Chiasso dalle 9.00 alle $16.00 \quad$ Fiduciaria

\section{Iscrizione}

Potete iscrivervi all'indirizzo sottoindicato oppure via internet sotto il sito www.fmhservices.ch.

\section{Anmeldung und Auskunft / Inscription et information}

FMH Consulting Services, Marianne Hochuli, Burghöhe 1, 6208 Oberkirch, Tel. 04192500 77, Fax 0419210586 oder via Internet unter www.fmhservices.ch.

\section{Annullierungsbedingungen}

Bei den Gratisseminaren (exkl. PraxiscomputerWorkshop) werden bei Abmeldungen oder Fernbleiben folgende Unkostenbeiträge erhoben:

- Fr. 50.- pro Person ab 14 Tage vor Seminarbeginn;

- Fr. 80.- pro Person ab 7 Tage vor Seminarbeginn oder Fernbleiben.

\section{Conditions d'annulation}

Pour les séminaires gratuits (à l'exclusion des séminaires sur informatique au cabinet médical), un montant est perçu pour absence ou annulation. Il est de:

- fr. 50.- par personne dans les 15 jours avant;

- fr. 80.- par personne dans les 7 jours avant le début du séminaire. 